\title{
Antithrombin III expression predicts acute kidney injury in elderly patients with sepsis
}

\author{
YUN XIE ${ }^{*}$, RUI TIAN* , WEI JIN* , HUI XIE, JIANG DU, ZHIGANG ZHOU and RUILAN WANG \\ Department of Critical Care Medicine, Shanghai General Hospital, \\ Shanghai Jiao Tong University School of Medicine, Songjiang, Shanghai 201600, P.R. China
}

Received June 13, 2019; Accepted October 18, 2019

DOI: $10.3892 /$ etm.2019.8305

\begin{abstract}
Elderly people represent the age group most frequently affected by acute kidney injury (AKI). The potential of Antithrombin III (ATIII) level for predicting AKI among elderly patients with sepsis is yet to be elucidated. Therefore, the purpose of the present study was to evaluate the ability of ATIII to predict AKI nondevelopment and prognosis in elderly patients with sepsis, in an intensive care unit (ICU). The present study was retrospective and included 107 elderly patients with sepsis who had been admitted to ICUs between October 2015 and March 2018. An assessment of renal function was performed daily by measuring serum creatinine $(\mathrm{Cr})$ level and urine output, and ATIII level was obtained within $48 \mathrm{~h}$ of sepsis diagnosis. Among all enrolled patients, 29 (27.1\%) developed AKI. ATIII expression was a predictor of AKI nondevelopment [Area under the curve (AUC)-Receiving operator characteristic $(\mathrm{ROC})=0.729$; sensitivity, 0.700 ; specificity, 0.714], and the ATIII/Creatine ratio was also a predictor of AKI nondevelopment (AUC-ROC $=0.971$; sensitivity, 0.900; specificity, 1). The accuracy of ATIII (AUC-ROC $=0.681$; sensitivity, 0.802; specificity, 0.542) and ATIII/Cr (AUC-ROC $=0.804$; sensitivity, 0.596; specificity, 0.875) in predicting survival was intermediate. However, the ATIII serum level was able to accurately predict AKI nondevelopment in elderly patients with sepsis, who were admitted to ICUs. Patients were divided
\end{abstract}

Correspondence to: Dr Ruilan Wang or Dr Zhigang Zhou, Department of Critical Care Medicine, Shanghai General Hospital, Shanghai Jiao Tong University School of Medicine, 650 New Songjiang Road, Songjiang, Shanghai 201600, P.R. China

E-mail: wangyusun@hotmail.com

E-mail: pollyerik2004@163.com

*Contributed equally

Abbreviations: APACHE II, Acute Physiology and Chronic Health Evaluation II score; SOFA, Sepsis-related Organ Failure Assessment; COPD, chronic obstructive pulmonary disease; ICU, intensive care unit; AKI, acute kidney injury; CRRT, continuous renal replacement therapy; ATIII, Antithrombin III; KDIGO, Kidney Disease Improving Global Outcomes

Key words: antithrombin III, acute kidney injury, biomarker into low- and high-ATIII groups using either $66.95 \%$ or $55.7 \%$ as cut-off values, both of which were used for further analysis. By comparison, the ICU stay was significantly lower in the high-ATIII group $[\mathrm{P}=0.020(69.95 \%)$ and $0.049(55.7 \%)]$ and off mechanical ventilation time, off continuous renal replacement therapy time and survival time were significantly higher in the high ATIII group [P=0.049, 0.048, and 0.014, respectively (66.95\%); and $\mathrm{P}=0.041,0.036$, and 0.021 , respectively (55.7\%)]. The current study indicated that ATIII serum level predicts AKI in elderly patients with sepsis, and that low ATIII levels predicted a poorer prognosis.

\section{Introduction}

Sepsis is defined as infection resulting in multiple organ dysfunction (1). Acute kidney injury (AKI) is a common early complication of sepsis, with an incidence ranging from $11-42 \%$ (1). In addition, the occurrence of AKI in patients with sepsis indicates poor prognosis and significantly increased mortality $(1,2)$. The elderly are the primary age group affected by AKI (3). Therefore, early detection of sepsis-induced AKI and timely treatment may improve the clinical prognosis of elderly patients with sepsis.

The mechanism by which sepsis induces AKI is yet to be elucidated. However, the inflammatory response to sepsis is considered to serve a role in kidney injury. Inflammatory responses in sepsis initiates the release of pro-inflammatory cytokines into the circulation, which results in systemic inflammatory responses (2). Multiple studies have demonstrated the associations between inflammatory factors and kidney oxygenation, metabolism, inflammation and function, where these factors promote leukocyte aggregation, which may result in renal inflammation (4-7). In addition, clinical studies have also suggested that novel biomarkers are required in order to detect AKI prior to creatinine and urine volume assessments (8), which with high susceptibility, is also a predictor of prognosis (8).

ATIII serves a vital role in anticoagulation and influences anti-inflammatory effects (8-10). Studies in animal models have indicated that ATIII exhibits a protective effect on the kidney, primarily via its anti-inflammatory activity (9-11). Furthermore, endogenous ATIII dysfunction exacerbates renal injury in animal models. In clinical studies, low ATIII levels increase the incidence of AKI in a number of patients $(12,13)$. 
However, further studies are needed to validate the protective effects of ATIII in sepsis-induced AKI. Although $\mathrm{Cr}$ and urine output can be used to diagnose AKI development, these assessments are often performed too late to identify AKI. The present study hypothesized that the occurrence of AKI in infectious elderly patients may be predicted by serum ATIII levels or a combination of ATIII and creatinine.

\section{Materials and methods}

Study population. In the current study, patients age $\geq 60$ years who presented with sepsis according to 'Sepsis 3.0' clinical criteria were included (14). The included data was collected from Shanghai General Hospital (Shanghai, China) between October 2015 and March 2018. The medical records of patients with sepsis who had been admitted to the intensive care unit (ICU) of Shanghai General Hospital were reviewed. The present study was approved by the institutional review board of the Shanghai General Hospital. Since the data published was retrospective and anonymized, the need for informed consent was waived.

Inclusion and exclusion criteria. In the present study, 'elderly' was defined as individuals ages $\geq 60$ years old, according to Chinese laws. AKI was defined as an increase in serum creatinine or decrease in urine output following the Kidney Disease Improving Global Outcomes (KDIGO) criteria (15). Patients were receiving no previous or ongoing treatment that may have effected the results of the current study. The inclusion criterion were: i) Patients of 'ICU' admission aged between 60 and 85 years that had ii) presented with sepsis, which was diagnosed according to the 'Survival Sepsis Campaign 2012' (14). The exclusion criteria were patients with: i) Stages 4 or 5 chronic kidney disease (creatinine clearance $<30 \mathrm{ml} / \mathrm{min}$ ); ii) a kidney transplant; iii) an ICU stay $<24 \mathrm{~h}$; and iv) a previous admission to the ICU with AKI. Patients with end-stage diseases (malignant tumour, leukaemia, cirrhosis, NYHA class IV, and severe COPD), a written 'do not resuscitate' form or a history of allergies to antibiotics or other drugs, were also excluded.

Baseline glomerular filtration rate was estimated using the Modification of Diet in Renal Disease study equation (16). Baseline creatinine was defined as the lowest serum creatinine value detected in the 6 months prior to AKI onset, or for those without this measurement, the lowest value achieved during hospitalization (in the absence of dialysis) (17). Complete data regarding the inclusion and exclusion processes are presented in Fig. 1.

Outcome. The primary outcome measured was AKI, and the secondary outcomes included use of continuous renal replacement therapy (CRRT), 28-day mortality, 90-day mortality, incidence of multiple organ dysfunction syndrome, shock, length of hospital stay (days), duration of mechanical ventilation (days) and use of a vasoactive agent.

Data collection. All data were retrospectively collected. Baseline data, including demographics, medical history and disease severity, were collected retrospectively for each patient by reviewing patients' medical records. Laboratory data were collected from the first day in the ICU. Vital signs, haemodynamic and laboratory data were collected each day during the ICU stay. Renal function was assessed daily according to creatinine levels and urine output. Serial Acute Physiology and Chronic Health Evaluation (APACHE) II score and Sequential Organ Failure Assessment (SOFA) score were calculated on the first day of admission to the ICU. AKI was categorized according to the KDIGO staging system 7 days after AKI diagnosis.

Blood samples were collected and tested for ATIII and $\mathrm{Cr}$ levels within the first $48 \mathrm{~h}$ of treatment of sepsis diagnosis in the ICU following admission. The samples were centrifuged $\left(3,000 \times \mathrm{g}, 37^{\circ} \mathrm{C}\right.$ and $10 \mathrm{~min}$ of centrifugation) and stored at $-80^{\circ} \mathrm{C}$ prior to subsequent analysis. ATIII was detected using human antithrombin III ELISA kit (cat. no. SY-01862; Shanghai win-win biotechnology company), and ATIII activity was measured via colorimetry. Following plasma incubation $\left(37^{\circ} \mathrm{C} ; 10 \mathrm{~min}\right)$ with heparin and thrombin in excess, residual thrombin was measured according to its amidolytic activity on the chromogenic substrate CBS 3447. Serum $\mathrm{Cr}$ was measured using an enzymatic colorimetric test based on the Jaffé reaction (alkaline picrate) (18).

Statistical analysis. Statistical analysis was performed using SPSS 13.0 (SPSS Inc.). Quantitative data are expressed as the mean \pm standard deviation. For data with a normal distribution, an independent sample t-test was used to compare the differences between two sets of data. For data that were not normally distributed, non-parametric tests were used to compare differences. $\chi^{2}$ test or Fisher's test were used for analysis of nominal data. A logistical regression analysis was used to evaluate the outcomes based on risk factors selected using univariate analysis. The diagnostic accuracy of ATIII levels in predicting AKI and mortality were assessed by calculating the area under the receiver operating characteristic curve (AUC-ROC). AUC-ROC analysis was performed by comparing patients with AKI to those without, and by comparing patient survival. The optimal cut-off values were defined as the highest values of sensitivity and specificity calculated via the AUC-ROC analysis. $\mathrm{P}<0.05$ was considered to indicate a statistically significant difference.

\section{Results}

Patient demographics and baseline characteristics. Study enrolment was undertaken between October 2015 and March 2018. Throughout this period, a total of 130 patients were selected for the present study. A total of 23 patients were excluded upon admission to the ICU, in accordance with the pre-defined exclusion criteria. Therefore, a total of 107 (82.3\%) patients were included for further analysis (Fig. 1).

Patient demographics and baseline characteristics are presented in Table I. In total, 65 patients were male $(60.7 \%)$, and $42(39.3 \%)$ patients were female. All 107 patients were included in the final data analysis (Fig. 1). The mean age was $69.76 \pm 4.773$ years, and the majority of patients had comorbidities $(87.8 \%)$. Hypertension, cardiovascular disease and diabetes mellitus were the most common comorbidities. The APACHE II score was $17.42 \pm 2.28$ and the SOFA score was $6.757 \pm 3.10$. The requirement for mechanical ventilation 
Table I. Patient demographics and clinical characteristics $(n=107)$ based on AKI.

\begin{tabular}{|c|c|c|c|c|c|}
\hline Clinicopathological characteristics & Non-AKI (n=78) & AKI (n=29) & P-value & $\chi^{2}$ value & Total $(n=107)$ \\
\hline Sex, \% male & $42(53.8)$ & $23(79.3)$ & $0.025^{\mathrm{a}}$ & 5.719 & $65(60.7)$ \\
\hline Age, years & $69.70 \pm 5.90$ & $69.90 \pm 5.83$ & 0.881 & 0.150 & $69.76 \pm 4.773$ \\
\hline \multicolumn{6}{|l|}{ Comorbidities, n (\%) } \\
\hline Hypertension & $36(46.1)$ & $22(75.9)$ & $0.008^{\mathrm{a}}$ & 7.516 & $58(54.2)$ \\
\hline Diabetes & $21(26.9)$ & $8(27.6)$ & 1.000 & 0.005 & $29(27.1)$ \\
\hline Immune diseases & $7(8.97)$ & $6(20.7)$ & 0.179 & 2.718 & $13(12.1)$ \\
\hline Cardiovascular disease & $40(51.3)$ & $24(82.7)$ & $0.004^{\mathrm{a}}$ & 8.714 & $64(59.8)$ \\
\hline Liver disease & $3(3.85)$ & $2(6.90)$ & 0.611 & 0.022 & $5(4.67)$ \\
\hline COPD & $16(20.5)$ & $3(10.3)$ & 0.268 & 0.881 & $19(17.7)$ \\
\hline Noradrenaline use, $\%$ & $32(41.0)$ & $23(79.3)$ & $\leq 0.001^{\mathrm{a}}$ & 12.404 & $55(51.4)$ \\
\hline Mechanical ventilation, $\mathrm{n}(\%)$ & $45(57.7)$ & $23(79.3)$ & $0.044^{\mathrm{a}}$ & 4.265 & $68(63.5)$ \\
\hline SOFA & $5.756 \pm 2.960$ & $9.448 \pm 3.611$ & $\leq 0.001^{\mathrm{a}}$ & 5.395 & $6.757 \pm 3.10$ \\
\hline APACHE II & $15.83 \pm 5.86$ & $21.69 \pm 7.04$ & $\leq 0.001^{\mathrm{a}}$ & 4.344 & $17.42 \pm 2.28$ \\
\hline Mortality & $24(30.8)$ & $16(89.6)$ & $0.026^{\mathrm{a}}$ & 5.378 & $40(37.4)$ \\
\hline Need for dialysis, n (\%) & $2(2.56)$ & $17(58.6)$ & $\leq 0.001^{\mathrm{a}}$ & 45.488 & $19(17.8)$ \\
\hline
\end{tabular}

AKI, acute kidney injury; COPD, chronic obstructive pulmonary disease; APACHE, Acute Physiology And Chronic Health Evaluation; SOFA, sequential organ failure assessment. Values are expressed as the mean \pm standard deviation or the median and interquartile range. ${ }^{\mathrm{a}} \mathrm{P}<0.05$.

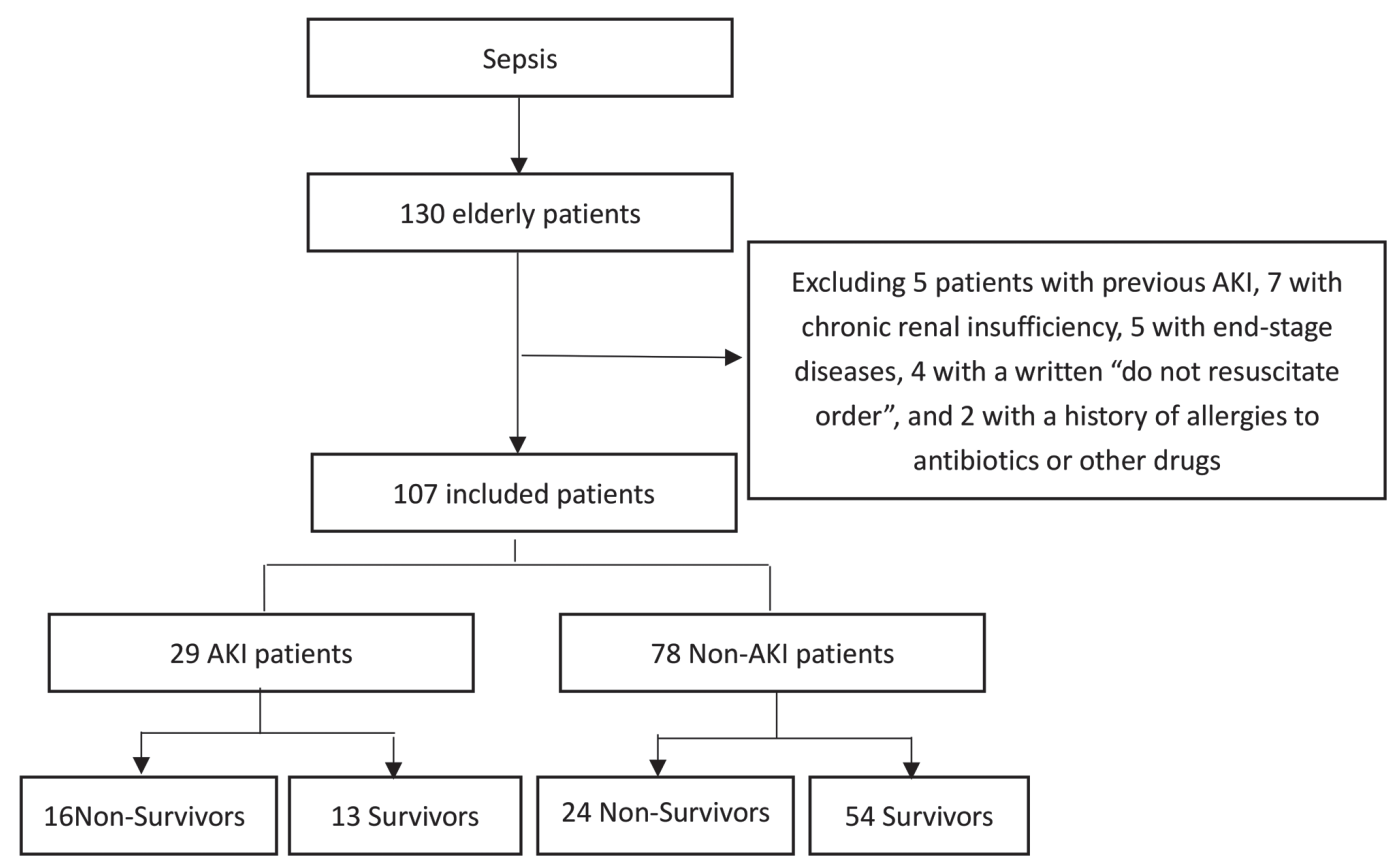

Figure 1. Flow chart describing the research method.

and noradrenaline use in the first $24 \mathrm{~h}$ after admission to ICU was 63.5 and $51.4 \%$, respectively. The primary source of infection was lung infection $(57.0 \%)$, followed by abdominal cavity $(43.0 \%)$ and urinary tract infection (24.0\%). A total of 29 patients $(27.1 \%)$ developed AKI, with the majority classified as KDIGO 3 (63.0\%). KDIGO 1 occurred in $11.1 \%$ and KDIGO 2 in $33.3 \%$ of patients. 'Need for dialysis' was indicated in $17.8 \%$, and the mortality rate was $37.3 \%$.

The characteristics of the non-AKI $(n=78)$ and AKI $(n=29)$ groups were similar with regards to age, diabetic status and comorbidity with immune disease, liver disease and COPD. Whereas patients who were male or had hypertension or cardiovascular disease were higher in patients with AKI, and mortality was higher in patients with AKI (89.6 vs. 30.8\%; $\mathrm{P}=0.026$ ). Table I indicates the clinical and laboratory characteristics of the population based on AKI. A significantly higher proportion of patients with AKI required mechanical ventilation $(79.3$ vs. $57.7 \%, \mathrm{P}=0.044)$, noradrenaline use (79.3 vs. 41\%; P<0.0001), APACHE II score $(21.69 \pm 7.04$ vs. $15.83 \pm 5.86 ; \mathrm{P}<0.0001)$ and SOFA score 
Table II. Patient demographics and clinical characteristics $(n=107)$ based on outcome.

\begin{tabular}{|c|c|c|c|c|c|}
\hline Clinicopathological characteristics & Non-Survivors $(n=40)$ & Survivors $(\mathrm{n}=67)$ & P-value & $\chi^{2}$ value & Total $(n=107)$ \\
\hline Sex, $\mathrm{n}$ (\% male) & $24(67.5)$ & $41(61.2)$ & 0.903 & 0.015 & $65(60.7)$ \\
\hline Age, years & $70.47 \pm 6.214$ & $69.33 \pm 5.634$ & 0.329 & 0.980 & $69.76 \pm 4.773$ \\
\hline \multicolumn{6}{|l|}{ Comorbidities, n (\%) } \\
\hline Hypertension & $22(55)$ & $36(53.7)$ & 0.899 & 0.016 & $58(54.2)$ \\
\hline Diabetes & $12(30)$ & $17(25.4)$ & 0.602 & 0.217 & $29(27.1)$ \\
\hline Immune diseases & $7(17.5)$ & $6(8.95)$ & 0.191 & 1.713 & $13(12.1)$ \\
\hline Cardiovascular disease & $26(65)$ & $38(56.7)$ & 0.398 & 0.715 & $64(59.8)$ \\
\hline Liver disease & $3(7.5)$ & $2(2.99)$ & 0.360 & 0.357 & $5(4.67)$ \\
\hline COPD & $8(20)$ & $11(16.4)$ & 0.639 & 0.220 & $19(17.7)$ \\
\hline Noradrenaline use, n (\%) & $37(92.5)$ & $18(26.9)$ & $\leq 0.001^{\mathrm{a}}$ & 43.193 & $55(51.4)$ \\
\hline Mechanical ventilation, n (\%) & $33(82.5)$ & $35(52.2)$ & $0.002^{\mathrm{a}}$ & 9.902 & $68(63.5)$ \\
\hline SOFA & $9.15 \pm 3.051$ & $5.328 \pm 3.012$ & $\leq 0.001^{\mathrm{a}}$ & 6.319 & $6.757 \pm 3.10$ \\
\hline APACHE II & $21.97 \pm 6.282$ & $14.70 \pm 5.359$ & $\leq 0.001^{\mathrm{a}}$ & 6.364 & $17.42 \pm 2.28$ \\
\hline AKI & $16(40)$ & $13(19.4)$ & $0.020^{\mathrm{a}}$ & 5.378 & $29(27.1)$ \\
\hline \multicolumn{6}{|l|}{ KDIGO, n (\%): } \\
\hline $\mathrm{I}$ & $2(5)$ & $1(1.49)$ & 0.554 & 0.131 & 32.80 \\
\hline II & $5(12.5)$ & $4(5.97)$ & 0.290 & 0.668 & $9(8.41)$ \\
\hline III & $10(25)$ & $7(10.4)$ & $0.046^{\mathrm{a}}$ & 3.969 & $17(15.9)$ \\
\hline Need for dialysis, n (\%) & $13(32.5)$ & $6(8.95)$ & $0.002^{\mathrm{a}}$ & 9.508 & $19(17.8)$ \\
\hline
\end{tabular}

AKI, acute kidney injury; COPD, chronic obstructive pulmonary disease; APACHE, Acute Physiology And Chronic Health Evaluation; SOFA, sequential organ failure assessment. ${ }^{a} \mathrm{P}>0.05$, no significant difference.Values are expressed as the mean and standard deviation or the median and interquartile range.

(9.448 \pm 3.611 vs. $5.756 \pm 2.960 ; \mathrm{P}<0.0001)$, compared with patients without AKI (Table I).

The characteristics of the non-survivor $(n=40)$ and survivor group $(n=67)$ were similar with regards to age, hypertension, diabetes, cardiovascular disease, immune disease, liver disease and COPD. AKI was more prevalent in non-survivors (40 vs. $19.4 \% ; \mathrm{P}=0.02$ ), and mortality was higher in AKI patients (55.17 vs. 30.77.9\%; P=0.02). Table I reveals the clinicopathological characteristics of the population based on hospital outcome. Compared with those without AKI, patients with AKI had a significantly higher need for mechanical ventilation ( 82.5 vs. $52.2 \% ; \mathrm{P}=0.002)$ and noradrenaline use $(92.5$ vs. $26.9 \%$; $\mathrm{P}<0.0001)$, and a higher APACHE II $(21.97 \pm 6.28$ vs. $14.70 \pm 5.36 ; \mathrm{P}<0.0001)$ and SOFA score $(9.15 \pm 3.05$ vs. $5.328 \pm 3.01 ; \mathrm{P}<0.0001)$, all of which were associated with poorer survival rates (Table II).

Multivariate analysis of AKI and mortality risk. Being male (OR, 0.140; 95\% CI, 0.030-0.643; $\mathrm{P}=0.011$ ), exhibiting hypertension (OR, 4.74; 95\% CI, 1.21-18.64; $\mathrm{P}=0.026)$, cardiovascular disease $(\mathrm{OR}, 5.36$; 95\% CI, 1.23-23.31; $\mathrm{P}=0.025)$ and ATIII (OR, 0.961; 95\% CI 0.93-0.99; $\mathrm{P}=0.005)$ were identified as independent risk factors for AKI in multivariate regression analysis (Table III).

ATIII values based on development of AKI and patient outcome. ATIII and ATIII/Cr were both higher in the non-AKI group compared with the AKI group: ATIII $(73.43 \pm 23.42$ vs. $54.01 \pm 25.46 \mathrm{~g} / 1 ; \mathrm{P}=0.003), \mathrm{ATIII} / \mathrm{Cr}$
Table III. Multivariate analysis of AKI and mortality risk $(\mathrm{n}=107)$.

\begin{tabular}{lccc}
\hline AKI & OR & $95 \%$ CI & P-value \\
\hline Male sex & 0.140 & $0.030-0.643$ & $0.011^{\mathrm{a}}$ \\
Hypertension & 4.744 & $1.207-18.643$ & $0.026^{\mathrm{a}}$ \\
Cardiovascular disease & 5.364 & $1.234-23.311$ & $0.025^{\mathrm{a}}$ \\
ATIII & 0.961 & $0.935-0.988$ & $0.005^{\mathrm{a}}$ \\
Risk of mortality & & & \\
Noradrenaline use & 1.129 & $1.00-1.275$ & 0.05 \\
Mechanical ventilation & 0.983 & $0.917-1.054$ & 0.633 \\
AKI & 2.406 & $0.977-5.926$ & 0.056 \\
ATIII & 0.980 & $0.958-1.002$ & 0.079 \\
\hline
\end{tabular}

AKI, acute kidney injury; ATIII, antithrombin III; OR, odds ratio; CI, confidence interval. ${ }^{\mathrm{a}} \mathrm{P}<0.05$.

( $1.10 \pm 0.75$ vs. $0.21 \pm 0.10 \mathrm{~g} / \mu \mathrm{mol} ; \mathrm{P}<0.0001)$. Similarly, ATIII and ATIII/Cr were higher in the survival group, compared with the non-survival group: ATIII (73.14 \pm 22.55 vs. $56.99 \pm 27.87 \mathrm{~g} / \mathrm{l}$; $\mathrm{P}=0.019), \mathrm{ATIII} / \mathrm{Cr}(1.06 \pm 0.81$ vs. $0.40 \pm 0.30 \mathrm{~g} / \mu \mathrm{mol} ; \mathrm{P}=0.0003$; Fig. 2).

ROC analysis of ATIII in elderly patients with sepsis with AKI vs.non-AKI. Fig. 3 indicates that ATIII was a predictor of AKI 


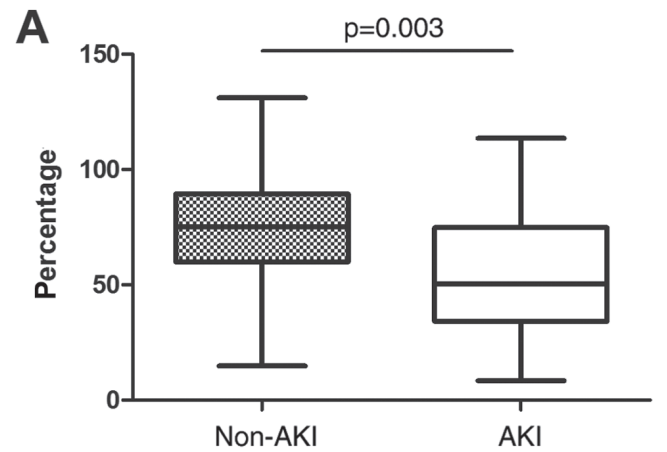

ATIII

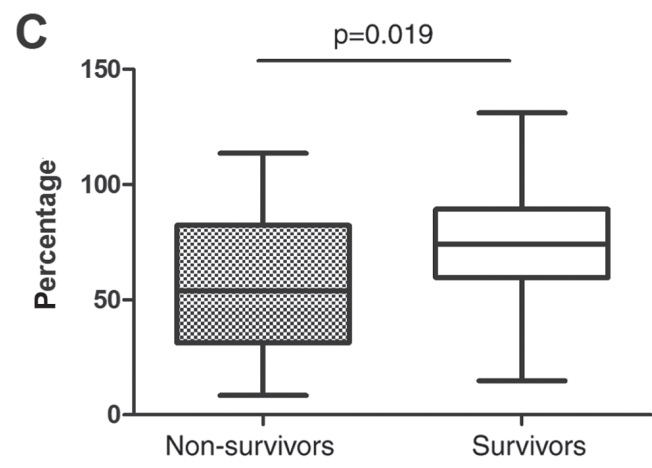

ATIII
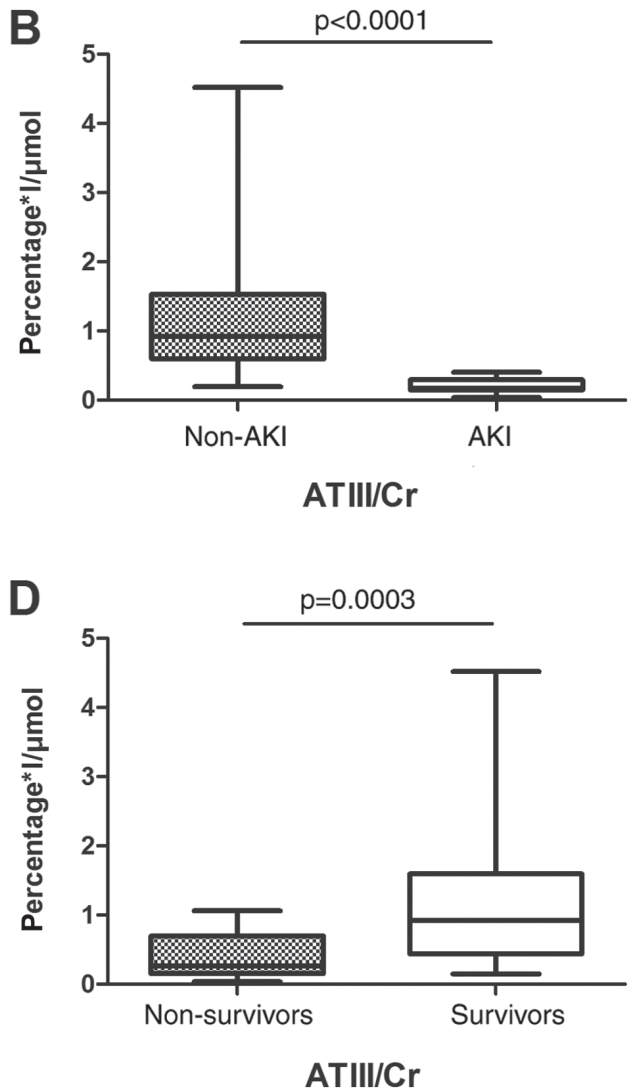

Figure 2. Serum ATIII and ATIII/Cr value in AKI. (A) Serum ATIII values based on development of acute kidney injury. (B) Serum ATIII/Cr values based on development of acute kidney injury. (C) Serum ATIII values based on outcome. (D) Serum ATIII/Cr values based on outcome. ATIII, antithrombin III; $\mathrm{Cr}$, creatine; AKI, acute kidney injury.

nondevelopment (AUC-ROC, 0.729; sensitivity 0.700; specificity, 0.714). The ATIII/Cr ratio was also a predictor of AKI nondevelopment (AUC-ROC, 0.971; sensitivity, 0.900; specificity, 1). The accuracy of ATIII (AUC/ROC, 0.681; sensitivity, 0.802; specificity, 0.542) and ATIII/Cr (AUC/ROC, 0.804; sensitivity, 0.596; specificity, 0.875) as predictors of survival was intermediate. ATIII and ATIII/Cr were accurate predictors of AKI nondevelopment at $48 \mathrm{~h}$ following admission to the ICU.

Regarding ATIII as a predictor of survival, the areas under the curve for ATIII and ATIII/Cr were 0.681 and 0.804 , respectively (Fig. 3C and D). The optimal cut-off value of each one had a sensitivity between 0.802 and 0.596 , and specificity between 0.542 and 0.875 (Table IV).

Patient outcome data with ATIII cut-off. The ATIII cut-off value in predicting AKI non-development in elderly patients with sepsis was $66.95 \mathrm{~g} / 1$ (Table IV). The ATIII cut-off used for predicting survival in elderly patients with sepsis was $55.70 \mathrm{~g} / 1$ (Table V). When patients were divided into a low ATIII group and high ATIII group using 66.95 or 55.70 as the cut-off value, the ICU stay was significantly lower in the high ATIII group ( $\mathrm{P}=0.020$ and 0.049 , respectively), and off mechanical ventilation time, off CRRT time, and survival time were significantly higher in the high ATIII group $(\mathrm{P}=0.049,0.048$, and 0.014 using 66.95 as the cut-off and $0.041,0.036$, and 0.021 using 55.70 as the cut-off value; Tables VI and VII).

\section{Discussion}

The present retrospective study aimed to evaluate the association between ATIII expression and certain clinical outcomes in elderly patients with sepsis, and revealed an association between decreased serum ATIII levels and AKI, mortality and other morbidities. It has been previously demonstrated that morbidity and mortality in sepsis is associated with decreased ATIII levels. A systemic review and meta-analysis of ATIII prospective randomized trials indicated no overall effect on mortality (19). However, the current study revealed that ATIII was associated with prediction of AKI in elderly patients with sepsis. Patients with a high expression of ATIII were indicated to have a shorter ICU stay, longer time without mechanical ventilation, longer time without CRRT and longer overall survival time.

It was discovered that $27.1 \%$ of elderly patients with sepsis developed AKI, and the majority developed AKI stage 3. This is consistent with a previous study, which reported that the incidence of AKI among geriatric patients ranged between 11 and $42 \%$ (1). The in-ICU mortality of geriatric patients with AKI (37.4\%) calculated in the present study, was similar to that reported in other studies (16-40\%) (3). Notably, being male, exhibiting hypertension, cardiovascular disease and having high serum ATIII level were all identified as predictors of AKI using logistic regression analysis. ATIII level was a predictor of AKI-nondevelopment (AUC-ROC $=0.729$; sensitivity 0.700; specificity, 0.714). The ATIII/Cr ratio was a predictor of 

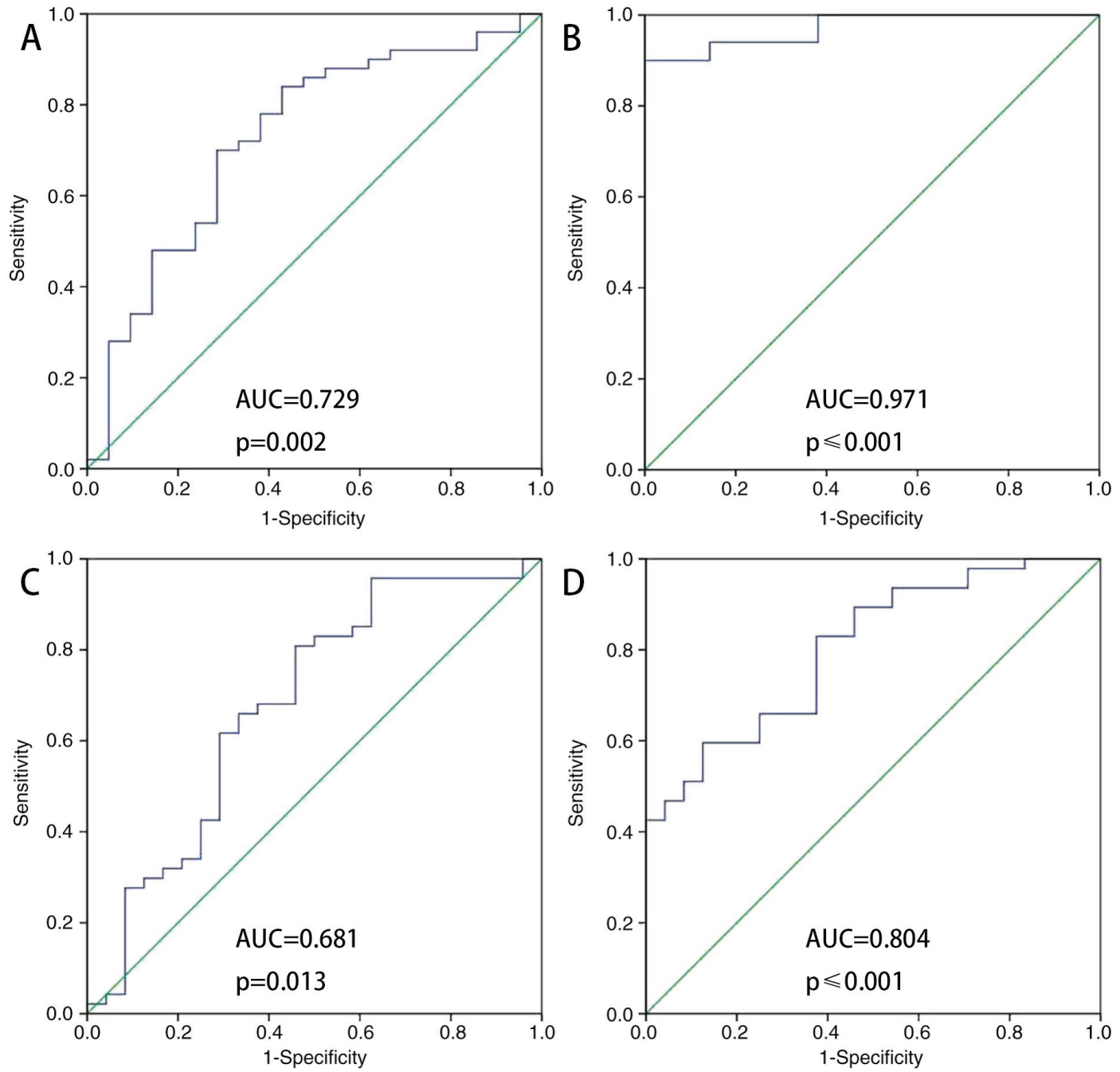

Figure 3. ROC analysis of ATIII in elderly patients with sepsis with AKI vs. non-AKI. (A) Analysis of ATIII measured within the first 48 h of admission to the ICU (B) Analysis of ATIII/Cr measured within the first $48 \mathrm{~h}$ of admission to the ICU. (C) Analysis of ATIII measured within the first $48 \mathrm{~h}$ of admission to the ICU (survivors vs. non-survivors). (D) Analysis of ATIII/Cr measured within the first $48 \mathrm{~h}$ of admission to the ICU (survivors vs. non-survivors). ROC, receiver operator characteristic; ATIII, antithrombin III; ICU, intensive care unit; Cr, creatine.

AKI-nondevelopment (AUC-ROC=0.971; sensitivity, 0.900; specificity, 1). The accuracy of ATIII and ATIII/Cr as predictors of survival was intermediate (AUC-ROC, 0.681 and 0.804 ; sensitivity of 0.802 and 0.596 , and specificity 0.542 and 0.875 , respectively). In the setting of the ICU, ATIII demonstrated accurate predictive value for AKI-nondevelopment in elderly patients with sepsis. The ATIII/Cr ratio was used because it makes more sense to measure both parameters simultaneously. This is because, decreased creatinine and increased ATIII predict the improvement of renal function injury; increased creatinine and decreased ATIII predict aggravation of severe renal damage; an increase in both creatinine and ATIII suggests that renal injury may not be caused by inflammation; and decreased creatinine suggests possible coagulation abnormalities $(4,9,10)$. This may also be the reason that the ATIII/Cr ratio exhibited higher significance (and a lower P-value) than ATIII.

To the best of our knowledge, this is the largest dataset analysed to determine the predictive potential of serum ATIII level on AKI development in patients with sepsis. There are articles have described the protective effect of ATIII on the kidneys, but these were studies conducted on animals $(12,20)$. Katayama et al (21) revealed that the AUC-ROC of AT III was 0.618 (0.564-0.670); however, in the present study the AUC-ROC of ATIII was calculated to be 0.729 (0.596-0.861), close to sTM 0.758 (0.677-0.825). Furthermore, the AUC-ROC of ATIII/Cr was $0.971(0.940-1)$. To the best of our knowledge, the prognostic value of ATIII level in the prediction of AKI in elderly patients with sepsis has not previously been elucidated. ATIII has been revealed as a promising single, universal predictive methodology for sepsis with an AKI diagnosis. In addition, further studies on ATIII will be further studied in the future and may help to improve evaluation of prognosis in patients with sepsis-associated renal injury, in addition to helping clinicians diagnose AKI more quickly and accurately. ATIII is gradually being introduced into clinical work in the setting of ICUs, however, there are also multiple challenges for its use, including a lack of universal standards for results analysis or guidelines for results interpretation. The early detection of AKI in sepsis is still challenging, requiring a combination of clinical judgement and other objective workups. 
Table IV. ATIII sensitivity and specificity in predicting AKI nondevelopment in elderly septic patients $(\mathrm{n}=107)$.

\begin{tabular}{lcccccc}
\hline & AUC-ROC & P-value & Cut-off value & Sensitivity & Specificity & $95 \%$ CI \\
\hline ATIII & 0.729 & $0.002^{\mathrm{a}}$ & 66.95 & 0.700 & 0.714 & $0.596-0.861$ \\
ATIII/Cr & 0.971 & $\leq 0.001^{\mathrm{a}}$ & 0.421 & 0.900 & 1 & $0.940-1$ \\
\hline
\end{tabular}

AKI, acute kidney injury; ATIII, antithrombin III; OR, odds ratio; CI, confidence interval. ${ }^{\mathrm{a}} \mathrm{P}<0.05$.

Table V. ATIII sensitivity and specificity in predicting survival of elderly septic patients $(n=107)$.

\begin{tabular}{lcccccc}
\hline & AUC-ROC & P-value & Cut-off value & Sensitivity & Specificity & $95 \%$ CI \\
\hline ATIII & 0.681 & $0.013^{\mathrm{a}}$ & 55.7 & 0.802 & 0.542 & $0.541-0.821$ \\
ATIII/Creatinine & 0.804 & $\leq 0.001^{\mathrm{a}}$ & 0.758 & 0.596 & 0.875 & $0.702-0.906$ \\
\hline
\end{tabular}

ATIII, antithrombin III; CI, confidence interval. ${ }^{a} \mathrm{P}<0.05$.

Table VI. Important clinical events of patients with ATIII.

\begin{tabular}{lcccr}
\hline Days & ATIII $\geq 66.95 \% \mathrm{n}=41$ & ATIII $<66.95 \% \mathrm{n}=28$ & P-value & $\chi^{2}$-value \\
\hline HLOS & $14.87 \pm 10.62$ & $18.40 \pm 15.14$ & 0.361 & -0.922 \\
ICU hospital stay & $9.81 \pm 5.78$ & $20.80 \pm 15.89$ & $0.020^{\mathrm{a}}$ & -2.599 \\
Duration of no vasoactive drug use & $26.36 \pm 4.48$ & $16.87 \pm 13.29$ & 0.088 & 1.940 \\
No mechanical ventilation time & $25.09 \pm 4.61$ & $13.12 \pm 14.12$ & $0.049^{\mathrm{a}}$ & 2.310 \\
No CRRT time & $26.46 \pm 5.13$ & $15.50 \pm 12.76$ & $0.048^{\mathrm{a}}$ & 2.297 \\
Survival time & $24.73 \pm 6.51$ & $18.78 \pm 10.99$ & $0.014^{\mathrm{a}}$ & 2.572 \\
\hline
\end{tabular}

ICULOS, intensive care unit length of stay; HLOS, hospital length of stay; CRRT, continuous renal replacement therapy; AKI, acute kidney injury; ATIII, antithrombin III; OR, odds ratio; CI, confidence interval. ${ }^{\mathrm{P}}<0.05$.

Table VII. Important clinical events of patients with ATIII.

\begin{tabular}{lcccr}
\hline Days & ATIII $\geq 55.7 \% \mathrm{n}=47$ & ATIII $<55.7 \% \mathrm{n}=22$ & P-value & $\chi^{2}$-value \\
\hline HLOS & $15.33 \pm 10.12$ & $18.18 \pm 17.85$ & 0.623 & -0.505 \\
ICU hospital stay & $10.44 \pm 6.08$ & $22.73 \pm 18.00$ & $0.049^{\mathrm{a}}$ & -2.225 \\
Duration of no vasoactive drug use & $26.50 \pm 4.30$ & $15.28 \pm 13.52$ & 0.072 & 2.134 \\
No mechanical ventilation time & $25.17 \pm 4.41$ & $11.28 \pm 14.17$ & $0.041^{\mathrm{a}}$ & 2.521 \\
No CRRT time & $26.58 \pm 4.91$ & $13.71 \pm 12.66$ & $0.036^{\mathrm{a}}$ & 2.579 \\
Survival time & $24.36 \pm 6.97$ & $17.95 \pm 11.32$ & $0.021^{\mathrm{a}}$ & 2.446 \\
\hline
\end{tabular}

ICULOS, Intensive care unit length of stay; HLOS, Hospital length of stay; CRRT, Continuous renal replacement therapy; ATIII, antithrombin. ${ }^{\mathrm{a}} \mathrm{P}<0.05$.

In the present study, ATIII exhibited a predictive effect on kidney injury caused by sepsis. Low ATIII levels are associated with an increased risk of AKI in patients with sepsis. In rat models, ATIII improves kidney injury prognosis primarily via the reduction of inflammation, apoptosis and oxidative stress. In addition, ATIII may serve an anti-inflammatory role by inhibiting the release of both inflammatory cytokines, and chemical kinase-mediated infiltration of immune cells (12). Therefore, ATIII may serve a predictive, preventative and protective role in sepsis-induced AKI. ATIII serine protease inhibitors not only represent major coagulant factor inhibitors in vivo, but also exhibit numerous clinical anti-inflammatory properties, including in disseminated intravascular coagulation and sepsis $(9,22)$. In addition to inhibiting inflammatory 
cytokines produced by endothelial cells (23), the mechanisms underlying the ATIII anti-inflammatory effect include increased synthesis and secretion of prostaglandin, inhibition of neutrophil roll and adhesion (24) and prevention of platelet activity (9).

ATIII does not appear to have a significant advantage over other biomarkers (including NGAL, KIM-1 and cystatin C) already used in the clinic. However, the limitations of the current study may have contributed to this; the retrospective design, small population size poor follow-up of long-term survival mean further studies with a larger sample size and improved study design may help further validate the findings. Nevertheless, ATIII represents a promising biomarker for renal injury secondary to sepsis, especially in hospitals where NGAL, KIM-1 and cystatin C cannot be detected.

The results of the present study indicated that ATIII can predict AKI in septic elderly patients, guide clinical management and reduce mortality. Clinics that adopt new methods, including ATIII assessment for patients with sepsis to assess the aim of personalized and precision therapy, may be able to utilize ATIII to predict AKI quickly and guide treatment. As a promising biomarker, ATIII may change conventional management of patients with sepsis.

In conclusion, the present study indicated that ATIII predicts AKI in elderly patients with sepsis. Therefore, lower serum ATIII levels may predict a poorer prognosis. The findings of the present study can lead to new ideas on ATIII and AKI prevention. Further studies are needed to confirm the role of ATIII administration in predicting AKI and increasing survival in patients with sepsis.

\section{Acknowledgements}

Not applicable.

\section{Funding}

The present study was supported by grants from the Important and Weak Subject Construction Project of Shanghai Health and Family Planning System (grant nos. 2016ZB0205 and 2016ZB0205, respectively), Shanghai Science and Technology Committee Scientific and Technological Support Project (grant nos. 18411950600 and 18411950602 , respectively), Clinical Research Innovation Plan of Shanghai General Hospital (grant no. CTCCR-2016B01) and Wu Jieping Medical Foundation (grant no. 320.6750.18546).

\section{Authors' contributions}

YX, RT, WJ, HX, JD, ZZ and RW contributed to the study conception and design, analysis and interpretation of data; drafting of the article and critical revision for important intellectual content. All the authors have read and approved the final manuscript.

\section{Ethics approval and consent to participate}

Ethics approval was obtained from Shanghai General Hospital Institutional Review Board [approval no. (2018)KY004].

\section{Availability of data and materials}

All data generated or analysed during this study are included in this published article.

\section{Patient consent for publication}

No applicable.

\section{Competing interests}

The authors declare that they have no competing interests.

\section{Authors' information}

Department of Critical Care Medicine, Shanghai General Hospital, Shanghai Jiao Tong University School of Medicine, 650 New Songjiang Road, Songjiang, Shanghai, 201600, China.

\section{References}

1. Godin M, Murray P and Mehta RL: Clinical Approach to the patient with AKI and Sepsis. Semin Nephrol 35: 12-22, 2015.

2. Gomez H, Ince C, De Backer D, Pickkers P, Payen D, Hotchkiss J and Kellum JA: A unified theory of sepsis-induced acute kidney injury: Inflammation, microcirculatory dysfunction, bioenergetics, and the tubular cell adaptation to injury. Shock 41: 3-11, 2014.

3. Cao Y and Wang RH: Associations among metabolism, circadian rhythm and age-associated diseases. Aging Dis 8: 314-333, 2017.

4. Wen X, Peng Z and Kellum JA: Pathogenesis of acute kidney injury: Effects of remote tissue damage on the kidney. Contrib Nephrol 174: 129-137, 2011.

5. Post EH, Kellum JA, Bellomo R and Vincent JL: Renal perfusion in sepsis: From macro- to microcirculation. Kidney Int 91: 45-60, 2017.

6. Regueira T, Andresen M, Mercado M and Downey P: Physiopathology of acute renal failure during sepsis. Med Intensiva 35: 424-432, 2011.

7. Kaifei W, Sheling X, Kun X, Peng Y, Wanxue H and Lixin X: Biomarkers of sepsis-induced acute kidney injury. BioMed Res International 2018: 1-7, 2018.

8. Lentini P, de Cal M, Clementi A, D'Angelo A and Ronco C: Sepsis and AKI in ICU patients: The role of plasma biomarkers. Crit Care Res Pract 2012: 856401, 2012.

9. Levy JH, Sniecinski RM, Welsby IJ and Levi M: Antithrombin: Anti-inflammatory properties and clinical applications. Thromb Haemost 115: 712-728, 2016.

10. Lu Z, Cheng D, Yin J, Wu R, Zhang G, Zhao Q, Wang N, Wang F and Liang M: Antithrombin III protects against contrast-induced nephropathy. EBioMedicine 17: 101-107, 2017.

11. Zhao Q, Yin J, Lu Z, Kong Y, Zhang G, Zhao B and Wang F: Sulodexide protects contrast-induced nephropathy in Sprague-Dawley rats. Cell Physiol Biochem 40: 621-632, 2016.

12. Wang F, Zhang G, Lu Z, Geurts AM, Usa K, Jacob HJ, Cowley AW, Wang N and Liang M: Antithrombin III/SerpinC1 insufficiency exacerbates renal ischemia/reperfusion injury. Kidney Int 88: 796-803, 2015.

13. Lu Z, Wang F and Liang M: SerpinC1/Antithrombin III in kidney-related diseases. Clin Sci (Lond) 131: 823-831, 2017.

14. Seymour CW, Liu VX, Iwashyna TJ, Brunkhorst FM, Rea TD, Scherag A, Rubenfeld G, Kahn JM, Shankar-Hari M, Singer M, et al: Assessment of clinical criteria for sepsis: For the third international consensus definitions for sepsis and septic shock (Sepsis-3). JAMA 351: 762-774, 2016.

15. Abdel-Rahman EM and Okusa MD: Effects of aging on renal function and regenerative capacity. Nephron Clin Prac 127: 15-20, 2014. 
16. Levey AS, Bosch JP, Lewis JB, Greene T, Rogers N and Roth D: A more accurate method to estimate glomerular filtration rate from serum creatinine: A new prediction Equation. Modification of Diet in Renal Disease Study Group. Ann Intern Med 130: 461-470, 1999.

17. Siew ED, Matheny ME, Ikizler TA, Lewis JB, Miller RA, Waitman LR, Go AS, Parikh CR and Peterson JF: Commonly used surrogates for baseline renal function affect the classification and prognosis of acute kidney injury. Kidney Int 77: 536-542, 2010.

18. Liu WS, Chung YT, Yang CY, Lin CC, Tsai KH, Yang WC, Chen TW, Lai YT, Li SY and Liu TY: Serum creatinine determined by Jaffe, Enzymatic method, and isotope dilution-liquid chromatography-mass spectrometry in patients under hemodialysis. J Clin Lab Anal 26: 206-214, 2012.

19. Allingstrup M, Wetterslev J, Ravn FB, Møller AM and Afshari A: Antithrombin III for critically ill patients: A systematic review with meta-analysis and trial sequential analysis. Intensive Care Med 42: 505-520, 2016

20. Kong Y, Yin J, Cheng D, Lu Z, Wang N, Wang F and Liang M: Antithrombin III Attenuates AKI following acute severe pancreatitis. Shock 49: 572-579, 2018.
21. Katayama S, Nunomiya S, Koyama K, Wada M, Koinuma T, Goto Y, Tonai K and Shima J: Markers of acute kidney injury in patients with sepsis: The role of soluble thrombomodulin. Crit Care 21: 229, 2017

22. Wang F, Peng C, Zhang G, Zhao Q, Xuan C, Wei M and Wang N: Delayed kidney injury following coronary angiography. Exp Ther Med 12: 530-534, 2016.

23. Souter PJ, Thomas S, Hubbard AR, Poole S, Römisch J and Gray E: Antithrombin inhibits lipopolysaccharide-induced tissue factor and interleukin- 6 production by mononuclear cells, human umbilical vein endothelial cells, and whole blood. Crit Care Med 29: 134-139, 2001.

24. Ostrovsky L, Woodman RC, Payne D, Teoh D and Kubes P: Antithrombin III prevents and rapidly reverses leukocyte recruitment in ischemia/reperfusion. Circulation 96: 2302-2310, 1997.

(i) () () This work is licensed under a Creative Commons

EY No AD Attribution-NonCommercial-NoDerivatives 4.0 International (CC BY-NC-ND 4.0) License. 